\title{
Impairment and activity limitation changes in assisted living facility residents over the course of one year- A pilot study
}

\author{
Sarah Larsen ${ }^{1}$, Rebecca Luedeker ${ }^{1}$, Carlos Morgan ${ }^{1}$, Christina Taylor ${ }^{1}$, Heather Braden ${ }^{1 *}$, Mansoo Ko ${ }^{2}$ and Heidi Moyer ${ }^{1}$ \\ ${ }^{1}$ Department of Physical Therapy, Angelo State University, ASU Station \#10923, San Angelo, TX, USA \\ ${ }^{2}$ Department of Physical Therapy, University of Texas Medical Branch, 301 University Boulevard, Galveston, TX, USA
}

\begin{abstract}
Background and purpose: When the elderly begin planning for a safe future, they may choose to move to an assisted living facility. Assisted living facilities (ALF) are a popular choice for senior living, but whether residents maintain their independence and movement ability over time in assisted living facilities has not been studied. The purpose of this study is to examine the changes in impairments and activity limitations in residents of ALF over the course of one year.

Participants: Fifteen residents from Brookdale South ALF and Sagora ALF, over the age of 65 years, volunteered to participate in the study.

Methods: Participants completed a series of outcome measures including: the standardized Mini-Mental State Examination (MMSE), gait speed, range of motion (ROM) for ankle dorsiflexion and plantarflexion, Timed Up and Go (TUG) test, handgrip strength, Five Time Sit To Stand Test (5XSST), Functional Independence Measure (FIM) scores, and manual muscle testing (MMT) of the hip, knee, and ankle. Measurements were obtained in the spring of 2015 and 2016. Statistical analysis was completed with SPSS Version 21 using paired-samples t-tests and Wilcoxon Sum Rank tests to compare the impairments and activity limitation changes over the course of a year.

Results: A significant decrease in left knee flexion strength, left hip adduction strength, right and left grip strength, right ankle plantarflexion ROM, and TUG speed over the course of a year was found. The participants demonstrated a significant increase in right dorsiflexion strength.

Discussion and conclusion: In ALF, individuals demonstrate baseline impairments and activity limitations that get worse over the course of a year. The elderly in this setting may experience a limited benefit from physical therapy to decrease the development of impairments and activity limitations for improved participation and quality of life.
\end{abstract}

\section{Introduction}

In 2014, the U.S. Department of Health and Human Services calculated an elderly population of 46.2 million individuals, equating to about one out of every seven American citizens [1]. It is predicted that the number of elderly will double by 2050 [2]. Additionally, there is a predicted increase of 27 million individuals using nursing facilities, home services, or alternative residential care options [3] in order to facilitate the expanding need of assistance in their later years of life [2]. According to the National Center for Assisted Living, more than 835,000 residents lived in assisted living facilities across the United States in 2016 [4]. ALF are ideal for individuals who wish to remain independent in most aspects of their life but need assistance with some activities of daily living such as eating, dressing, and self-care. These facilities also provide health-related services such as nursing and physical rehabilitation to older individuals [5].

It is well known that with increasing age, a decline in an individual's bodily functions and structures, otherwise known as impairments, are expected. In the elderly population, a combination of psychological and physical changes diminishes the ability of these individuals to complete daily tasks [6]. When an individual demonstrates decreased ability to complete functional tasks, a routine of sedentary behavior and task avoidance develops leading to a poorer quality of life [6]. This decline in function could be explained by the limited amount of physical activity offered to the elderly or their limited participation [7]. At least $30 \%$ of individuals who enter a residential care facility show a decline in muscle strength, muscle power, cardiovascular function, and neuromuscular response times [8]. If not addressed, these impairments could lead to more general activity limitations, further decreasing their quality of life.

According to the literature, ALF residents have weaker hand muscles as compared to their community dwelling counterparts [9]. The health-related quality of life of these residents, as measured by such factors as vitality, physical functioning, general health, and bodily pain [10], have been shown to be related to hand muscle strength [11]. There is a strong association between handgrip strength and health decline in the elderly population as many functional tasks, such as dressing and eating, require the use of the hands. Furthermore, studies indicate that "handgrip strength predicts decline in function, psychological, and social health in the oldest old" and with weaker handgrip strength one

Correspondence to: Heather Braden, Professor, Department of Physical Therapy, Angelo State University, ASU Station \#10923, San Angelo TX, USA. Tel: 325-942-2581; Fax: 325-942-2548; E-mail: hbraden@angelo.edu

Received: October 01, 2017; Accepted: October 18, 2017; Published: October 21, 2017 
can anticipate an accelerated decrease in quality of activities of daily living (ADL) leading to increased dependency in older age [12,13].

Decreased muscle strength and ROM in the lower extremities predispose individuals to functional decline and disability [14]. Reductions in ankle ROM have been shown to decrease participation in life roles in older adults [15]. According to a study by Bok, et al, ankle ROM and strength decrease with age and correlate with decreased balance and increased risk of falls [16]. In addition, older men who have decreased strength in the lower extremities have been associated with a variety of impairments regarding their physical function, including an inability to perform ADLs and instrumental activities of daily living (IADLs) [17]. Ultimately, this inability to perform ADLs and IADLs independently and a fear of falling can contribute to a decreased quality of life for elderly individuals. ${ }^{12}$

Clinicians commonly use screening tools or standardized physical performance tests on the elderly population to predict healthrelated outcomes to identify older adults at high risk of poor health, functional limitations, and falls [18]. However, there are few studies which determine the common impairments that often appear over the course of a year in the ALF population. Furthermore, in this population, there are limited studies that examine the relationship of these impairments and the impact on the residents' ability to execute a task or activity. The assessment tools implemented in this study aim to evaluate the functional mobility and impairments in assisted living facility residents. These assessment tools include: hand strength with a handgrip dynamometer, MMT with the Microfet2 ${ }^{\mathrm{TM}}$, ankle ROM with a goniometer, and TUG, walking speed, and the 5XSST with a stopwatch. The purpose of this report is to explore the results of the selected outcome measures that indicate areas of impairments and functional decline within the ALF population over the course of one year. With a better understanding of the trends in functional mobility, activity limitations, and impairments of this population, physical therapists will better be able to provide meaningful assessments and treatments addressing their specific needs and reducing adverse events. This can lead to the ability to age in place with improved quality of life.

\section{Methods}

\section{Participants}

Participants were recruited via means of convenience sampling at two ALF in TX. The facilities used for the study population were Brookdale South and Sagora ALF. Inclusion criteria to participate in this study consisted of the following: 60 years of age or older and ambulatory at least 15 feet modified independently or independently. Exclusion criteria included the following: a past medical history of stroke, diagnosis of terminal illness likely to result in death within a year, IV lines, inability to legally consent to procedures, and hospitalization within the 6 weeks prior to participation in the research study.

\section{Procedures}

The research study was approved by the Angelo State University Institutional Review Board and performed under the supervision of a licensed physical therapist. Researchers contacted staff of Brookdale South and Sagora of San Angelo via email and phone to set up times for both ALF to gather health information and to recruit participants for the research study. Prior to information sessions and with management permission, flyers with a brief overview of the study were posted in the hallways of the facilities. Management was asked to encourage residents who could potentially meet inclusion criteria to come to the information session. During the information sessions, the researchers explained the purpose, inclusion and exclusion criteria, as well as the process of the study to the attending residents. A brief demonstration of the tools used and tests performed for the study was conducted. At the end of the information session, the researchers emphasized the fact that participation was optional and those who chose to participate were given an opportunity to set up an appointment that best suited their schedule. To increase the number of participants for the study, the researchers asked management to recommend other residents who did not attend the information session and would potentially be interested in participating in the study. Management recommended additional residents, and they received the same information as those at the information session in a one-on-one setting. Those who chose to participate were added to the schedule. Door-to-door recruitment was also incorporated with management permission. Each subject signed a consent form prior to participation in the study.

Equipment used for the research study included: two firm, identical chairs without arm support, handheld dynamometers for strength testing, grip strength dynamometer, stopwatch, gait belt, goniometer, cones, and a tape measure to mark distances in ambulatory tests. Both dynamometers were calibrated. Gait speed and TUG test setup can be seen in Figure 1.

Data was gathered by two pairs of physical therapy researchers. The researchers used a standardized procedure to measure all participants as verified in an initial training session. Researchers testing the same components of the study followed the same order of testing and methods of measurements for consistency and accuracy.

Clinical impairments tested were the following: bilateral lower extremity (B LE) strength via handheld dynamometer including hip, knee, and ankle, ankle dorsiflexion (DF) and plantar flexion (PF) active range of motion (AROM), and hand grip strength.

Activity limitations tested were as follows: gait speed, Timed Up and Go Test (TUG), and Five Times Sit to Stand Test (5XSST). Data for the following tests and measures was gathered in this particular order:

- Vital Signs - Resting blood pressure and heart rate were gathered.

- Assistive device use assessment - Researchers noted the use and type of an assistive device by the participant. FIM status was evaluated as an observational measure during the ambulatory test.

- MMSE - The researchers asked a series of questions that assessed cognitive impairment. This was performed once for each visit. This was performed to ensure participant ability to follow directions.

- Gait speed - The participant was asked to walk at a self-selected pace for 6 meters with an untimed 2 meter acceleration phase and a 2 meter deceleration phase. The participant was cued with the words "go" and "stop."

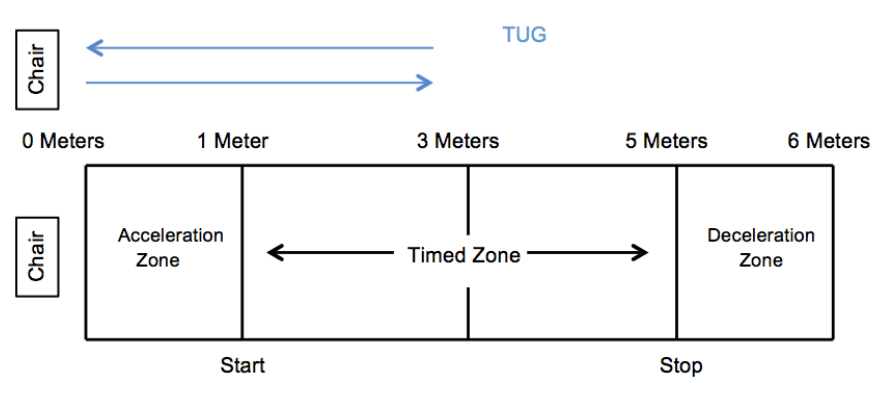

Figure 1. TUG and Gait Speed Testing. 
- TUG - The participant started in the seated position and was instructed to stand up, walk 3 meters, turn around a cone, walk back to the chair, and sit down as quickly and safely as possible. Timing started when they initiated the movement and stopped when they returned to the starting position, with their buttocks at the back of the chair.

- Full lower extremity MMT screen (hip, knee, and ankle) using a Microfet $2^{\mathrm{TM}}$ handheld dynamometer - Participants were seated in a firm, armless chair and stabilized/supported by the researcher(s) for comfort and ease of testing. Two attempts were allowed on each leg and tested in the following order: hip flexion, hip extension, hip abduction, hip adduction, knee flexion, and knee extension. Shoes were then removed for ankle dorsiflexion and plantarflexion tests. The average of the two attempts was calculated and used in the final compilation of results.

- Degree of ankle plantarflexion/dorsiflexion active ROM - Seated in the chair, socks and shoes were removed, the participant straightened the knee of the leg being tested and dorsiflexed/plantarflexed the foot. Measurements were gathered twice on each ankle. The average of the measurements was used in the final compilation of the results.

- Isometric handgrip strength - The participant was asked to squeeze the handgrip dynamometer as strongly as possible twice with each hand. The average of the measurements was used in the final compilation of the results.

- Five Time Sit to Stand Test (5XSST) - The participant was seated in the armless chair and asked to stand up and sit down fully at a swift pace five times. The participant was timed from the initiation of movement to completion in the seated position after the fifth chair stand.

Each researcher in the pair performed a specific set of tests with consistent wording, paired with demonstration. Each participant wore a safety/gait belt in the event that assistance was needed during the study. The researchers stood near the participant at stand by assist (SBA) for all tests with no constant contact that would impact test results. One researcher assessed vitals, assistive device use, MMSE, ROM of the ankle using a goniometer, and grip strength. The other researcher tested muscle strength, gait speed, TUG, and 5XSST. Demographic data for age, height, weight, gender, marital status, current number of oral medications, ethnicity, current active diagnoses, number of falls occurring 6 months prior to start of study, number of falls occurring over the course of the study, recent hospitalizations, and admission date to ALF were collected. Additionally, the participants were tested in the spring of 2015 for initial data and in the spring of 2016 for final data collection. The same tests and measures were taken by the same pairs of researchers.

\section{Statistical analysis}

Data was analyzed using SPSS version 21 (IBM, Chicago, IL). Statistical significance was considered at $\mathrm{p}<0.05$. Researchers ran a Shapiro-Wilk test of normality and then utilized paired-samples t-tests for the dependent variables whose data showed normality. Because the study is a within-subject design, researchers used a Wilcoxon Rank Sum Test for the measures that failed to meet normality. Data was evaluated to determine if there was a correlation between impairments and function in 2015 and again for 2016.

\section{Results}

Fifteen participants (mean of 88.5 years old) consented to complete the study over the course of a year. Figure 2 outlines the participant
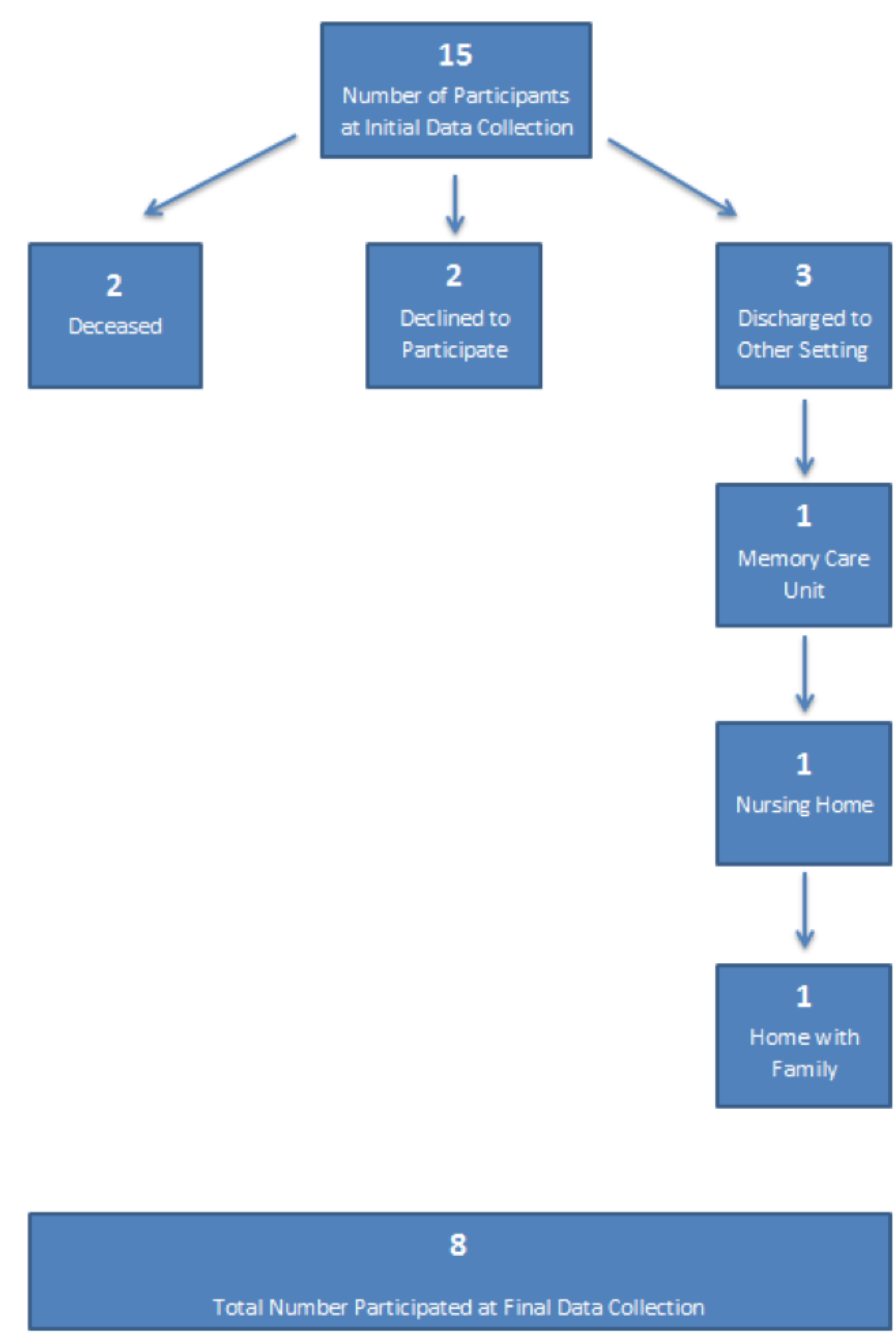

Figure 2. Participant attrition rate.

attrition rate. A total of 15 participants were tested initially; however, over the course of a year due to participant wellness issues, 7 were not able to complete the final session: 3 moved to another residence, 2 declined to participate, and 2 expired.

All variables met the normality requirements except for MMT of the left hip abduction strength, ROM of right ankle dorsiflexion, TUG, and gait speed. Calculated paired t-test outcomes are depicted in Table 1. When comparing initial data to final data, there was a significant difference in the scores for the following measures: left knee flexion strength decreased $\mathrm{t}(7)=2.752, \mathrm{p}=0.028$; left hip adduction strength decreased $\mathrm{t}(7)=3.7354, \mathrm{p}=0.012$; right plantarflexion $\mathrm{ROM}$ decreased $\mathrm{t}(7)=2.508 ; \mathrm{p}=0.041$ : right grip strength decreased $\mathrm{t}(7)=5.409, \mathrm{p}=$ 0.001 ; left grip strength decreased $\mathrm{t}(7)=3.551, \mathrm{p}=0.009$; and right ankle dorsiflexion strength increased $t(7)=-2.923, p=0.022$.. The Wilcoxon Rank Sum Test demonstrated significant slowing in TUG times from year 1 to year $2(Z=-2.370, p=0.017)$. Table 2 includes the significant correlations between impairment and function at initial data collection, and Table 3 includes the same final data collection.

\section{Discussion}

Overall, the results of this study reveal significant changes at the impairment level over the course of the year in the form of the 
Table 1. Comparing initial data to data collected 1 year later.

\begin{tabular}{|c|c|c|c|c|c|}
\hline \multirow[b]{2}{*}{ Paired Samples Test } & \multicolumn{2}{|c|}{ Paired Differences } & \multirow[b]{2}{*}{$\mathbf{t}$} & \multirow[b]{2}{*}{ df } & \multirow{2}{*}{$\begin{array}{c}\text { Sig. } \\
\text { (2-tailed) }\end{array}$} \\
\hline & Mean & \begin{tabular}{|c|} 
Std. \\
Deviation
\end{tabular} & & & \\
\hline Mini Mental Status Exam & 1.50000 & 2.20389 & 1.925 & 7 & .096 \\
\hline Right dorsiflexion strength & -4.06875 & 3.93718 & -2.923 & 7 & $.022 *$ \\
\hline Left dorsiflexion strength & -1.38750 & 4.69435 & -.836 & 7 & .431 \\
\hline Right plantarflexion strength & 2.72500 & 12.38896 & .622 & 7 & .554 \\
\hline Left plantarflexion strength & 10.87500 & 15.33244 & 2.006 & 7 & .085 \\
\hline Right knee flexion strength & 5.15000 & 6.90776 & 2.109 & 7 & .073 \\
\hline Left knee flexion strength & 6.95000 & 7.14263 & 2.752 & 7 & $.028 *$ \\
\hline Right knee extension strength & .94375 & 8.65693 & .308 & 7 & .767 \\
\hline Left knee extension strength & 5.81875 & 12.68500 & 1.297 & 7 & .236 \\
\hline Right hip extension strength & -4.40625 & 8.24666 & -1.511 & 7 & .174 \\
\hline Left hip extension strength & 2.96875 & 4.88562 & 1.719 & 7 & .129 \\
\hline Right hip flexion strength & 2.65000 & 7.30205 & 1.026 & 7 & .339 \\
\hline Left hip flexion strength & -.11875 & 8.36540 & -.040 & 7 & .969 \\
\hline Right hip adduction strength & 7.24875 & 13.40707 & 1.529 & 7 & .170 \\
\hline Left hip adduction strength & 7.80000 & 6.57729 & 3.354 & 7 & $.012 *$ \\
\hline Right hip abduction strength & .90625 & 10.25272 & .250 & 7 & .810 \\
\hline Left hip abduction strength & 1.49375 & 14.16861 & .298 & 7 & .774 \\
\hline Right dorsiflexion range of motion & 6.43750 & 9.59329 & 1.898 & 7 & .099 \\
\hline Left dorsiflexion range of motion & 6.93750 & 15.89348 & 1.235 & 7 & .257 \\
\hline Right plantarflexion range of motion & 9.31250 & 10.50149 & 2.508 & 7 & $.041^{*}$ \\
\hline Left plantarflexion range of motion & 3.81250 & 4.94930 & 2.179 & 7 & .066 \\
\hline Right grip strength & 5.56250 & 2.90858 & 5.409 & 7 & $.001 *$ \\
\hline Left grip strength & 5.65625 & 4.50582 & 3.551 & 7 & $.009^{*}$ \\
\hline 5 Times sit-to-stand (seconds) & -3.94250 & 18.36854 & -.607 & 7 & .563 \\
\hline Number of falls & -.25000 & .70711 & -1.000 & 7 & .351 \\
\hline
\end{tabular}

Table 2. Initial data collection - within year significant results.

\begin{tabular}{|c|c|c|}
\hline & Correlation & Significance \\
\hline Falls to TUG & .887 & $.003^{*}$ \\
\hline Left hip abduction strength to 5xSST & .803 & $.016^{*}$ \\
\hline Right hip flexion strength to 5xSST & .738 & $.036^{*}$ \\
\hline Right hip flexion strength to falls & .728 & $.041^{*}$ \\
\hline Right hip adduction to 5xSST & .847 & $.008^{*}$ \\
\hline Left hip adduction to 5xSST & .746 & $.034^{*}$ \\
\hline * Denotes statistical significance at $\mathrm{p}<0.05$. & \\
\hline
\end{tabular}

Table 3. Final data collection - within year significant results.

\begin{tabular}{|c|c|c|}
\hline & Correlation & Significance \\
\hline Right hip flexion strength to falls & .794 & $.019^{*}$ \\
\hline Right plantarflexion ROM to falls & -.856 & $.007^{*}$ \\
\hline Gait speed to weight & -.828 & $.011^{*}$ \\
\hline Gait speed to right plantarflexion strength & .975 & $.047^{*}$ \\
\hline Gait speed to left hip extension strength & -.794 & $.019^{*}$ \\
\hline Gait speed to TUG & .975 & $.000^{*}$ \\
\hline * Denotes statistical significance at $\mathrm{p}<0.05$ & \\
\hline
\end{tabular}

following: decreased left knee flexion and hip adduction strength, decreased bilateral grip strength, decreased right plantarflexion ROM, and increased right dorsiflexion strength. The only activity limitation measure with significance over the course of the year was the TUG test. Per the within-year results, some of the aforementioned areas of decreased strength and ROM were correlated with increased levels of disability as measured by functional tests such as the TUG, gait speed, and the 5XSST. Poor performance on these functional tests is correlated with higher levels of disability and prevalence of falls. Disability and falls leads to increased healthcare costs in addition to decreased independence and quality of life [19,20]. A single nonfatal fall cost
$\$ 9,780$ on average in 2015 and approximately $\$ 31.3$ billion total for all nonfatal falls per year [19]. Identifying risk factors and preventing falls have great monetary value, as it allows the individual to save financial resources and use them more efficiently.

\section{Significant clinical impairment measures: Handheld dynamometer/strength and active range of motion}

Over the course of the year, the decrease in the plantarflexion ROM could be explained by a decrease in the amount of daily ambulatory activities, leading to the decreased utilization of the full ROM at the ankle. Residents primarily walk within the facility on level floors, minimizing the need for ankle compensation strategies like one would need if ambulating on grass, gravel, or over curbs in the community. Plantarflexion range is important as it promotes a normal gait pattern. If there is a decrease in this range, the individual may start to develop less effective and efficient gait habits, thereby decreasing the distance and speed they can walk independently.

In addition, the decreases in left knee flexion and left hip adduction strength could pose additional mobility issues in the future. This decline in strength could be attributed to leg dominance or a decrease in activities that require the use of these muscles, resulting in deconditioning. As it relates to functional mobility, lower extremity muscle strength is important for the maintenance of the proper gait cycle in addition to the ability to perform functional activities, such as standing up from a chair or getting into and out of the bathtub. Inability to perform daily functional tasks decreases the safety of daily living and the individual's perceived independence [21,22].

Right ankle dorsiflexion strength significantly improved in the ALF residents over the duration of the year. This finding was an unexpected result. Functionally, dorsiflexion strength is important in gait mechanics, as it is a vital component that assists with foot clearance during the swing phase of the gait cycle. If an individual is unable to perform the necessary dorsiflexion secondary to a pathology, the risk of unsuccessful toe clearance and falls increases $[23,24]$. The falls could inevitably lead to increased health problems, decreased independence, and decreased quality of life $[12,17]$. The ALF offered seated exercises that included active dorsiflexion, and residents are asked to walk to the dining room for 3 meals each day, which can be 150 ' to $1000^{\prime}$, depending on the location of their apartment entrance from dining.

Left and right handgrip strength significantly decreased over the year. This decline in grip strength could be explained by the residents not participating in activities that required the use of significant grip strength. In the general population, grip strength is likely to be more heavily utilized through common activities such as: steering a car, carrying heavy grocery bags, gardening, wringing a towel, or engaging in manual labor. Most of these activities, if not all, are typically unnecessary tasks for those in ALF. Furthermore, with aging, there is a degree of natural muscle decline to be expected [8]. There are various reasons a decline in grip strength is concerning, including the fact that grip strength is necessary to pick up objects, open doors or jars, and safely use an assistive device. Grip strength is imperative to daily activities and research has shown a strong correlation to grip strength and quality of life [11]. Grip strength is said to have a strong correlation with functional performance ${ }^{24}$ and is a predictor of functional decline, nursing home placement, and even mortality [25]. As one example, it has been shown that female ALF residents that fall generally have weaker handgrip strength as compared to their female ALF non-faller counterparts [26]. In contrast to these findings, another study indicates that grip strength is, at best, only weakly related to functional capacity 
(as measured by TUG and the Barthel Index) [27]. However, there is concern about the interpretability of the Barthel Index because changes in function may not be apparent due to the variability of the scoring system [28]. In addition, reliability has been questioned when used with the older population [29]. Therefore, the current evidence available could suggest that interventions designed by physical therapists to improve grip strength would be beneficial for the daily functioning and well-being of ALF residents.

\section{Significant activity limitation measure: Timed Up and Go Test (TUG)}

According to the Wilcoxon Rank Sum Test results, there was a significant increase in time to complete the TUG from year 1 to year 2. Research demonstrates that older adults who complete the TUG in 20 seconds or less have been shown to be independent in transfer tasks and tend to have adequate walking speed times $(0.5 \mathrm{~m} / \mathrm{s})$ in community ambulation [21]. After a year, TUG times in the ALF population significantly increased, indicating higher risk for falls and/ or more assistance with ambulation. This population would benefit from further examination and intervention.

These results suggest that significant changes in impairments and one activity limitation occur over the course of one year in ALF. Assisted living facility residents could benefit from physical therapy interventions to prevent this decline. As these impairments eventually become large enough to impact functional mobility, it is important to strength and ROM early in the process. If the changes in function over the course of a year persist with no interventions, they would likely lead to adverse events and need for placement in a more expensive and dependent setting for their continuum of care.

\section{Limitations}

The limitations of this study include potential human error and inconsistency in the inter-rater reliability with ankle MMT and ROM measurements. Inter-rater reliability for handheld dynamometer for MMT ranges from good to high according to a study by Bohannon in which researchers tested six different muscles of 30 participants [30]. Another study shows a strong intra-rater test-retest correlation for MMT using the handheld dynamometer in community-dwelling elderly fallers [31]. It demonstrates that the measurement of MMT with the dynamometer is a helpful tool to utilize, but the population of the study does not directly correlate with the elderly ALF population. As for goniometry, specifically of the ankle, inter-rater reliability was found to be 0.7 [32]. Demographics within the study were another limitation. Participants were Caucasian and primarily females. This research, therefore, could most accurately be applied and related toward elderly Caucasian females in an ALF. However, according to the CDC National Survey of Residential Care Facilities Survey, approximately $70 \%$ of residents are female and approximately $91 \%$ are Caucasian [33]. Therefore, while the demographics of the study do not fully represent the small amount of diversity seen on a larger scale, the data can be applied to the overwhelming majority of the national population of those residing in an ALF, as far as gender and ethnicity are concerned. Finally, the most concerning limitations to the study were the attrition rate and the number of residents tested. A larger number of participants to test and retest over the year would have been ideal. In addition, increased participant numbers would make the research study more applicable to a larger population.

\section{Future studies}

Future studies could reproduce the current study and document hand and foot dominance to determine if there is a correlation to falls or determine if there is a difference in the rate of strength decline between dominant and non-dominant sides over the course of a year. Future studies may benefit from either having a single person taking certain measurements or testing the inter-rater reliability of those chosen to perform the measurements. This will help reduce the likelihood of measurement error. Finally, there would be great value in performing the study in a larger demographic region to have access to a larger population sample. The larger population sample for the research study would be helpful to accurately determine the changes in the $\mathrm{AL}$ population over the course of a year to represent ALF residents as a whole, therefore enabling physical therapists to better serve individuals in this setting.

\section{Conclusion}

In conclusion, research has shown that changes in function occur with aging and have been associated with decline in quality of life [6]. This study demonstrates that there is a development of clinical impairments and a single activity limitation measure that was tested in ALF residents over the course of a year. It may be beneficial to provide physical therapy for individuals in this setting to help prevent, screen for, and specifically address strength and ROM impairments in attempt to prevent more observable activity limitations from subsequently occurring. Regardless of when implemented, interventions can slow the progression of impairments that may preserve independence, promote safe mobility, and improve quality of life for residents in assisted living facilities.

\section{Acknowledgements}

We would like to thank the assisted living facility staff and families for making this study possible.

\section{Declaration of interest}

The authors report no declarations of interest.

\section{Funding}

Grant Support: Research Enhancement Grant, Angelo State University, 2016-17entitled Impairment and Activity Limitation Changes in Assisted Living Facility Residents Over the Course of One Year.

\section{References}

1. Aging Statistics. Aoaaclgov. 2016. Available at: http://www.aoa.acl.gov/Aging Statistics/index.aspx. Accessed September 10, 2016.

2. Crocker T, Young J, Forster A, Brown L, Ozer S, et al. (2013) The effect of physica rehabilitation on activities of daily living in older residents of long-term care facilities: systematic review with meta-analysis. Age and Ageing 42: 682-688. [Crossref]

3. Harris-Kojetin L, Sengupta M, Park-Lee E, Valverde R, Caffrey C, et al. (2016) LongTerm Care Providers and services users in the United States: data from the Nationa Study of Long-Term Care Providers, 2013-2014. Vital Health Stat 3: x-xii. [Crossref]

4. National Center for Assisted Living: Residents. Ahcancal.org. 2017. Available at: https://www.ahcancal.org/ncal/facts/Pages/Residents.aspx. Accessed May 1, 2017.

5. Fields NL, Koenig T, Dabelko-Schoeny H (2012) Resident transitions to assisted living: a role for social workers. Health Soc Work 37: 147-154. [Crossref]

6. Alcock L, O’Brien T, Vanicek N (2015) Age-related changes in physical functioning correlates between objective and self-reported outcomes. Physiotherapy 101(2): 204 213. [Crossref]

7. Resnick B, Galik E, Gruber-Baldini A, Zimmerman S (2011) Testing the effect of function-focused care in assisted living. J Am Geriatr Soc 59: 2233-2240. [Crossref]

8. Peri K, Kerse N, Robinson E, Parsons M, Parsons J, et al. (2007) Does functionally based activity make a difference to health status and mobility? A randomised controlled trial in residential care facilities (The Promoting Independent Living Study; PILS). Age and Ageing 37: 57-63. [Crossref] 
9. Vaiserman A (2004) Developmental epigenetic programming of caste-specific differences in social insects: an impact on longevity. Curr Aging Sci 7: 176-86.

10. Sayer AA, Syddall HE, Martin HJ, Dennison EM, Roberts HC, et al. (2006) Is grip strength associated with health-related quality of life? Findings from the Hertfordshire Cohort Study. Age Ageing 35: 409-415. [Crossref]

11. Kanwar A, Singh M, Lennon R, Ghanta K, McNallan SM, et al. (2013) Frailty and health-related quality of life among residents of long-term care facilities. J Aging Health 25: 792-802. [Crossref]

12. Suzuki M, Ohyama N, Yamada K, Kanamori M (2002) The relationship between fear of falling, activities of daily living and quality of life among elderly individuals. Nursing \& Health Sciences 4(4):155-161.

13. Taekema D, Gussekloo J, Maier A, Westendorp R, de Craen A. Handgrip strength as a predictor of functional, psychological and social health. A prospective populationbased study among the oldest old. Age and Ageing 39: 331-337.

14. Rantanen T, Guralnik JM, Foley D, Masaki K, Leveille S, et al. (1999) Midlife hand grip strength as a predictor of old age disability. JAMA 281: 558-560. [Crossref]

15. Beauchamp M, Jette A, Bean J (2016) Leg and Trunk Impairments Predict Participation in Life Roles in Older Adults: Results from Boston RISE. The Journals of Gerontology 71: 663-669.

16. Bok S-K, Lee TH, Lee SS (2013) The Effects of Changes of Ankle Strength and ROM According to Aging on Balance. Annals of Rehabilitation Medicine 37(1): 10-16.

17. Hairi N, Cumming R, Sambrook P (2016) Loss of muscle strength, mass (sarcopenia), and quality (specific force) and its relationship with functional limitation and physical disability: the Concord Health and Ageing in Men Project. Journal of The American Geriatrics Society 58(11): 2055-2062.

18. Viccaro L, Perera S, Studenski S (2011) Is Timed Up and Go Better Than Gait Speed in Predicting Health, Function, and Falls in Older Adults?. Journal of the American Geriatrics Society 59(5): 887-892.

19. Burns E, Stevens J, Lee R (2016) The direct costs of fatal and non-fatal falls among older adults-United States. Journal of Safety Research 58: 99-103.

20. Akase R, Tori K, Nojiri S, Yamanaga H (2015) Risk factors for falls in the disabled elderly that maintain independence in activities of daily living. Physiotherapy 44.

21. Shumway-Cook A, Brauer S, Woollacott M (2000) Predicting the probability for falls in community-dwelling older adults using the Timed Up \& Go Test. Phys Ther 80: 896-903. [Crossref]
22. Sahin H, Erkal S (2015) Assessment of the Activities of Daily Living of the Elderly in Reference to the Thunder Bay Index. Turkish Journal of Geriatrics 18: 217-223.

23. Mills PM, Barrett RS, Morrison S (2008) Toe clearance variability during walking in young and elderly men. Gait Posture 28: 101-107. [Crossref]

24. Martien S, Delecluse C, Boen F, Seghers J, Pelssers J, et al. (2015) Is knee extension strength a better predictor of functional performance than handgrip strength among older adults in three different settings? Arch Gerontol Geriatr 60: 252-258. [Crossref]

25. Al Snih S, Markides KS, Ray L, Ostir GV, Goodwin JS (2002) Handgrip strength and mortality in older Mexican Americans. J Am Geriatr Soc 50: 1250-1256. [Crossref]

26. Sai AJ, Gallagher JC, Smith LM, Logsdon S (2010) Fall predictors in the community dwelling elderly: a cross sectional and prospective cohort study. $J$ Musculoskelet Neuronal Interact 10: 142-150.

27. Tietjen-Smith T, Smith S, Martin M, Henry R, Weeks S, et al. (2006) Grip Strength in Relation to Overall Strength and Functional Capacity in Very Old and Oldest Old Females. Physical \& Occupational Therapy in Geriatrics 24: 63-78.

28. Webster L, Groskreutz D, Grinbergs-Saull A, Howard R, O’Brien JT, et al. (2017) Development of a core outcome set for disease modification trials in mild to moderate dementia: a systematic review, patient and public consultation and consensus recommendations. Health Technol Assess 21: 1-192. [Crossref]

29. Sainsbury A, Seebass G, Bansal A, Young JB (2005) Reliability of the Barthel Index when used with older people. Age Ageing 34: 228-232. [Crossref]

30. Bohannon, Richard WA (1987) Williams Andrews. "Interrater reliability of hand-held dynamometry." Physical Therapy 67.6: 931-933.

31. Wang C, Olson S, Protas E (2002) Test-retest strength reliability: Hand-held dynamometry in community-dwelling elderly fallers. Archives of Physical Medicine and Rehabilitation 83: 811-815.

32. Martin RL, McPoil TG (2005) Reliability of ankle goniometric measurements: a literature review. J Am Podiatr Med Assoc 95: 564-572. [Crossref]

33. Who Lives in Assisted Living? - Assisted Living Facilities. Assistedlivingfacilities org. 2017. Available at: https://www.assistedlivingfacilities.org/resources/who-livesin-assisted-living/. Accessed May 1, 2017.

Copyright: (C2017 Larsen S. This is an open-access article distributed under the terms of the Creative Commons Attribution License, which permits unrestricted use, distribution, and reproduction in any medium, provided the original author and source are credited. 